\title{
Laparoscopic Management of Gall Bladder Polyp
}

\author{
Dr. Ashok Gajbhiye, Dr. Nithin Raj, Dr. Kuntal Surana \\ Study conducted at IGGMC, Nagpur
}

Objective: The objective of this study was to assess the outcome of patients managed laparoscopically with prediagnosis of gall bladder polyps.

Methods:

Study type: - Prospective.

Study duration: - Six year Jan 2008 to December 2013

Study Place:-IGGMC Nagpur

Sampling Frame: - Prediagnosed symptomatic cases of gall bladder polyp which are operated for the same.

Sample size: - Sample is taken from above sampling frame which were according to the below mentioned inclusion criteria.

Inclusion criteria:

1) Symptomatic patients having right hypochondriac discomfort.

2) Age more than 60 years.

3) Polyp diameter more than $6 \mathrm{~mm}$.

4) Sessile polyps.

5) Solitary polyps.

6) Associated gallstones and primary sclerosing cholangitis.

7) HbsAg positive patients.

Exclusion criteria:

1) Incidental asymptomatic finding.

2) Age less than 60 years.

3) Polyp size less than $6 \mathrm{~mm}$.

4) Cholesterol and inflammatory polyps.

5) Short pedunculated and multiple small polyps.

Result: Out of the 26 cases operated all were found to be benign on follow up. The mean age at diagnosis was 45.5 years and most of the cases were males. The mean diameter of the polyps was $6 \mathrm{~mm}$.

Conclusion: Out of the patients operated for Gall bladder polyp 24 patients after a period of follow up for 1 year were completely alright and 2 patients continued to have mild right hypochondriac discomfort. Hence laparoscopic management is a good option for the patients fulfilling the inclusion criteria.

Key Words: Gall bladder polyp, Laparoscopic

\section{Introduction:}

Lesions that project from the gallbladder wall into the gallbladder interior are called gallbladder polyps (GPs). In the majority of patients, diagnosis is an incidental finding of a routine abdominal ultrasound or following cholecystectomy for gallstones or biliary colic. Even though most of the gallbladder polyps are benign in nature, malignant polyps are present in some cases, and early detection and appropriate early measure is important for curative treatment and long-term survival. The primary goal in the management of gallbladder polyps is to prevent the development of gallbladder carcinoma.

The term polypoid lesions of the gallbladder represents a wide spectrum of findings. Gallbladder polyps are classified as benign or malignant. Benign GPs are subdivided into: pseudotumors (cholesterol polyps, inflammatory polyps; cholesterolosis and hyperplasia), epithelial tumors (adenomas) and mesenchymatous tumors (fibroma, lipoma, and hemangioma). Malignant GPs are gallbladder carcinomas. The poor prognosis of gallbladder carcinoma patients means it is important to differentiate between benign polyps and malignant or premalignant polyps.[1]

\section{Incidence Of Gallbladder Polyps}

The prevalence of gallbladder polyps was reported as $4.3-6.9 \%$ in general population. Polypoid gallbladder lesions include a variety of pathologic types.[2] Although there are some differences according to reports, the prevalence of polypoid lesions of the gall bladder are reported in $2-12 \%$ of cholecystectomy specimens, probably dependent on indications for cholecystectomy.[]ㅡ 
Age and gender seem to be notable factors for incidence of gallbladder polyps. In a study of 1558 patients with gallbladder polyps, the age at the time of diagnosis was 49 years. [4] In the majority of publications in which the ratio is calculated, the incidence is more prevalent in men.[1,5] The sex ratio and age at the time of diagnosis of gallbladder polyps was 1.15 to 1 (male to female).[4, $\underline{6}]$

Benign polyps

Malignant and premalignant polyps

$\begin{array}{cc}<10 \mathrm{~mm} & >10 \mathrm{~mm} \\ 25 & 1 \\ 0 & 0 \\ <50 \text { Years } & >50 \text { Years } \\ 19 & 7 \\ 0 & 0 .\end{array}$

Benign polyps

Malignant and premalignant polyps

\section{Risk factors for polyps}

Polyps of gall bladder are tumor-like lesions of this organ. Little has been known about factors associated with the occurrence of gallbladder polyps. The formation of gallbladder polyps is however associated with fat metabolism. Relationship between gallbladder polyps and family history of some diseases suggests to perform some genetic studies.[]]

In contrast to the well-known risk factors for gallstones, attempts to identify risk factors for developing gallbladder polyps have not shown any consistent relationship between formation of polyps and age, gender, obesity, or medical conditions such as diabetes. There is some literature to suggest an inverse relationship between gallbladder polyps and stones. It is hypothesized that polyps either mechanically disrupt the formation of stones or that polyps are harder to diagnose radiographically when stones are present. [8]

Patients with congenital polyposis syndromes such as Peutz-Jeghers and Gardner syndrome can also develop gallbladder polyps. A large retrospective analysis of risk factors for gallbladder polyps in the Chinese population identified chronic hepatitis B as a risk factor.[] ]

Proposed patient risk factors for malignant gallbladder polyps include age greater than 60, presence of gallstones, and primary sclerosing cholangitis. Polyp risk characteristics include a size greater than $6 \mathrm{~mm}$, solitary, and sessile.[8]

\section{Symptomatology}

The presenting symptoms of polypoid lesions of the gallbladder are nonspecific and vague, and in many cases asymptomatic. For such reason, polypoid lesions of the gallbladder are often detected incidentally.[3,9] However, there are some patients with gallbladder polyps which may suffer nausea, vomiting, and occasional pain in the right hypochondrium, due to intermittent obstructions caused by small fragments of cholesterol that become detached from the gallbladder mucosa. There are descriptions of polyps that protrude greatly obstructing the cystic canal or the primary biliary ducts, causing acute cholecystitis or obstructive jaundice, but these are very rare complications.[1]

Polyps are sometimes identified on transabdominal ultrasounds done for right upper quadrant pain. In the absence of other findings, the gallbladder polyp may be considered a source of biliary colic. If any symptoms are caused, most commonly right upper quadrant pain, nausea, dyspepsia, and jaundice are seen. However, most of the patients have gallstones; it is unclear whether the polyps were primarily driving the symptoms. There was no difference in presenting symptoms between patients with benign versus malignant polyps. In a large retrospective analysis found to have gallbladder polyps on abdominal ultrasound, $64 \%$ of these polyps were diagnosed during a work-up of unrelated illness. Twenty-three percent had abdominal symptoms, and $13 \%$ had elevated liver function tests. Cholesterol polyps may detach and behave clinically as a gallstone, causing biliary colic, obstruction, or even pancreatitis. There are also reports of gallbladder polyps causing acalculous cholecystitis or even massive hemobilia.[] $]$

Symptoms may be associated with pseudo-polyps such as a cholesterol polyp, inflammatory or hyperplastic polyp, which include indigestion, right upper quadrant pain, and discomfort, cholecystitis or gallbladder stone.[10] Metabolic syndrome has a close relationship with the development of cholesterol polyps

\section{Relation between gallbladder stones and gallbladder polyps}

The purpose of one study was to determine the clinical characteristics of subjects with gallbladder polyps and cholelithiasis compared with those with gallbladder polyps only. No significant difference in the mean interval change of polyp size during the follow-up period between the study and control groups was noted. A significantly higher proportion of examinees in the study group had attacks of acute cholecystitis compared with the control group. By multivariate logistic regression analysis, gallbladder wall thickening on initial ultrasonography (US) and the interval increase in the size of the gallbladder polyps were significant independent risk factors for cholecystectomy. No gallbladder cancer occurred during the follow-up period. There was, 
therefore, no significant difference in delta polyp size between the examinees with gallbladder polyps and cholelithiasis and those with gallbladder polyps only.[11]

Some patients who are confirmed to have polypoid lesions of the gallbladder through cholecystectomy are associated with gallstone. Symptoms were significantly associated with malignant polyp compared with benign polyp. The association of symptom and presence of associated gallstone was separately analyzed. Significant association of symptom to associated gallstone was not found. However, symptomatic cases tended to increase with the increase in polyp size. Therefore, a speculation can be made that symptom may be associated with the size of the polyp rather than the association of gallstone.[]]

\section{Imaging of gallbladder polyps}

The development and refinement of diagnostic imaging modalities such as computed tomography (CT), magnetic resonance imaging (MRI), and US and their widespread application have led to an increase in the coincidental diagnosis of gallbladder stones and gallbladder polyps. However, the appropriate management of these entities remains controversial.[12]

\section{Ultrasonography}

Abdominal ultrasound is looked upon as the best available exam for diagnosing gallbladder polyps, not only because of its accessibility and low cost, but also because of its good sensitivity and specificity. The polyps can be located, counted, and measured with ultrasound, and the three layers of the gallbladder wall and any abnormalities can be viewed.[1] The polyps appear as fixed, hyperechoic material protruding in to the lumen of the gallbladder, with or without an acoustic shadow. The sensitivity of abdominal ultrasound for diagnosis of gallbladder polyps is superior to both oral cholecystography and CT and good to distinguish a cholesterol polyp from an adenoma or an adenocarcinoma. A cholesterol polyp shows as a mass with similar echogenicity to the gallbladder wall and with no shadow cone. However, the distinction is difficult to make, and the status of polyps as benign or malignant cannot be determined with abdominal ultrasound alone.[1] Generally, polyps in the gallbladder are demonstrable on US, only when they are over $5 \mathrm{~mm}$ in diameter. Sonographic differentiation between benign and malignant polyps (and calculous disease) relies greatly on the size of a single nonmobile lesion within the gall bladder. A gallstone impacted within the gallbladder wall may be easily mistaken for a polyp on ultrasound scanning.[13]

However, abdominal ultrasound is often limited by the body habitus of the patient, and technical limitations can lead to intraobserver variability in interpretation. Abdominal ultrasound is generally considered the first of line study for making this diagnosis; it is by no means a definitive indicator of the presence of a gallbladder polyp or its malignant potential.[8] A retrospective study reported the symptoms probes were picking up small mucosal folds or misdiagnosing gallstones that did not have posterior shadowing or were immobile. Ultrasound can underestimate maximum diameter, and size assessments can be misleading. Ultrasound is therefore unable to reliably distinguish between nonneoplastic and neoplastic polyps.[9]

The measurement of lesion size by conventional US alone is inadequate for differentiating them. Cholesterol polyps of the gallbladder can readily be detected by abdominal ultrasound. They are composed of foamy histiocytes that contain cholesterol, are covered by a single layer of columnar cells similar to those lining the adjacent mucosa, and are considered benign.[14]

\section{The discrepancy between US and CT scanning}

The mean values for CT scanning tended to be smaller than for US. The discrepancies in maximum diameters between US and CT scanning were $6 \pm 4 \mathrm{~mm}$ in the cholesterol polyp group and $2 \pm 2 \mathrm{~mm}$ in the noncholesterol polyp group, and this difference was statistically significant.[10] In diagnosis of polypoid lesions of the gallbladder, the discovery rate of B-ultrasound is significantly higher than that of CT or cholecystography. Therefore, B-ultrasonography could be first used for the diagnosis of polypoid lesions of the gallbladder. However, CT could display local anatomic correlations of the liver, gallbladder, porta hepatis, and the other organs. Enhanced CT could improve the discovery rate of polypoid lesions of the gallbladder for CT.[15]

Small polypoid lesions of the gallbladder are easily detected by US, but accuracy based solely on US is controversial.[16] The accuracy of sonography for diagnosing polypoid lesions of the gallbladder was poor. Many of the small polyps seen on sonography most likely represented a stone embedded in the gallbladder wall or other abnormality.[1] $]$

\section{Three-dimensional ultrasonography}

Three-dimensional ultrasound (3DUS) diagnosis correlates well with two-dimensional ultrasound (2DUS) with regard to most gallbladder problems and could be sufficient as a stand-alone technique.[18] 


\section{High-resolution ultrasonography}

Endoscopic ultrasonography (EUS) was considered the most sensitive diagnostic modality for gallbladder polypoid lesions. However, the diagnostic accuracies of high-resolution ultrasound (HRUS) and EUS for the differential diagnosis of gallbladder polypoid lesions were comparable. In view of patient comfort and no requirement for sedation, it was considered that HRUS is likely to become an important diagnostic modality for the differential diagnosis and staging of gallbladder polypoid lesions and early gallbladder cancer.[19]

\section{Harmonic imaging}

The quality of images in the harmonic mode is better, and the walls of the gall bladder are more distinct. The polyps were therefore more evident on harmonic images, which are more precise measurements of the polyps. In the harmonic mode, the level of artifacts generated by the body wall is reduced and contrast resolution is increased due to reduction in noise level. The visualization of gallbladder is improved in the harmonic mode.[20]

\section{Contrast-enhanced ultrasonography}

Advances in conventional US, such as high-resolution US, have contributed to improved detection of polypoid gallbladder lesions. A galactose-based contrast agent was used in the US for differential diagnosis of polypoid gallbladder lesions.[2] When diffuse and branched types were considered indicative of cancer, accuracy was $85 \%$, sensitivity $100 \%$, and specificity $77 \%$. In gallbladder cancer, staining throughout the tumor was continuous, consistent with diffuse hypervascularity. In benign gallbladder polyps, staining was scattered with the flow image being uniform and small. Ultrasonographic contrast enhancement patterns therefore show characteristic associations with pathologic findings, serve as valuable adjuncts in the diagnosis of gallbladder diseases,[21] and differentiate gallbladder carcinoma from other polypoid gallbladder disease lesions.[22]

\section{Endoscopic ultrasonography (EUS)}

EUS is better than B-ultrasonography.[15] EUS has gained widespread use for the diagnosis of gastrointestinal malignancies, submucosal lesions of the gastrointestinal tract, and abnormalities seen on crosssectional imaging. [8] EUS is also recommended for further examination after conventional US, because images obtained are more distinct than with conventional US. Such images appear promising for distinguishing cholesterol polyps from other polyps, and the overall accuracy for differentiating neoplastic from non-neoplastic masses was reported as $91 \% .[2,8]$

Whether EUS alone can be used to determine a treatment strategy for gallbladder polyps is not clear. EUS alone is not sufficient in determining the course of treatment in polyps less than $10 \mathrm{~mm}$, but it may be more accurate than transabdominal ultrasound in determining whether gallbladder polyps are neoplastic.[8] Distinguishing between non-neoplastic, neoplastic, and potentially malignant lesions is a major diagnostic dilemma, and the therapeutic options for these lesions remain controversial. EUS is considered to be superior to conventional US for imaging gallbladder lesions, because EUS can provide high-resolution images of small lesions with higher ultrasound frequencies (7.5-12 MHz vs. 3.5-5 MHz).[8] Although EUS was more accurate than US, its accuracy for differentiating malignancy less than $1.0 \mathrm{~cm}$ was low.[23,24]

\section{Comparison with transabdominal ultrasonography}

Transabdominal ultrasonography (US) has made the detection of gallbladder polyps easier, but the differential diagnosis of polyps less than $20 \mathrm{~mm}$ remains difficult. EUS markedly improve the accuracy of the differential diagnosis of gallbladder polyps and is thought to play an important role in determining the treatment strategy for gallbladder polyps. $[25,26]$

\section{EUS scoring system}

A scoring system to predict neoplastic polyps of the gallbladder has been presented.[27] The total EUS score based on the coefficient of multivariate analysis was as follows: (maximum diameter in $\mathrm{mm}$ ) + (internal echo pattern score; where heterogeneous $=4$, homogeneous $=0)+($ hyperechoic spot score; where presence $=$ 5 , absence $=0$ ). According to EUS scoring system, the sensitivity, specificity, and accuracy for the risk of neoplastic polyps with scores of 12 or higher were $78 \%, 83 \%$, and $83 \%$, respectively.[28]

\section{Computed tomography}

Abdominal CT is incapable of detecting low density lesions, and its sensitivity for diagnosis of gallbladder polyps is not such satisfied, especially when gallbladder polyps were smaller than $10 \mathrm{~mm}$ in diameter, but it is useful for studying gallbladder carcinoma, anatomic correlations, and for investigating metastases of the ganglia.[1, 10] Advances in multidetector-row CT have increased its accuracy rate for the 
differential diagnosis of gallbladder polyps, and CT generally shows polypoid gallbladder carcinoma as an enhancing, intraluminal tissue mass denser than surrounding bile and can reliably identify neoplastic lesions.[2] In particular, helical CT may be helpful for evaluating small polypoid lesions of the gallbladder, and can differentiate neoplastic and nonneoplastic small polypoid lesions of the gallbladder and reliably identify the presence of neoplastic lesions that should be resected.[14] The size bigger than $1.5 \mathrm{~cm}$, sessile shape, and perception on unenhanced images are the main factors that differentiate neoplastic from nonneoplastic gallbladder polyps $1 \mathrm{~cm}$ or bigger.[29]

\section{Magnetic resonance imaging}

Magnetic resonance imaging has not been widely used to evaluate gallbladder diseases, having the disadvantages of poor spatial and contrast resolution. Among polypoid masses, malignant lesions demonstrated early and prolonged enhancements, while benign lesions showed early enhancement with subsequent washout.[2] It has reported that various malignant tumors may show high signal intensity on diffusion-weighted MR imaging (DWI), reflecting their high cellularity and/or their long relaxation time. Therefore, high b-value DWI may be useful for differentiating between benign and malignant polypoid gallbladder lesions.[30]

\section{Positron emission tomography}

It was presented a small case series of patients with gallbladder polyps that were correctly diagnosed preoperatively as benign or malignant with the use of positron emission tomography scanning with ${ }^{18} \mathrm{~F}$-labelled deoxyglucose.[]

\section{Intravenous cholecystography}

Intravenous cholecystography is a safe technique, but gallbladder polyps do not become sufficiently opaque.[1]

\section{Transpapillary approach}

Percutaneous transhepatic fine-needle aspiration and percutaneous transhepatic cholecystoscopy have been reported as precise diagnostic techniques in the evaluation of gallbladder polyps, but it is time consuming and poorly tolerated by patients. In addition, the diagnostic accuracy of endoscopic retrograde cholangiopancreatography is not satisfactory, because this can only show a filling defect in the gallbladder without delineating the surface of polypoid lesions.[2]

\section{Histopathology}

Histologic classification and identification of the nature of gallbladder polyps remain a dilemma. In fact, the classification of polyps was very confusing until the introduction made by Christensen and Ishak in 1970's. Subsequently, a comprehensive review by the Armed Forces Institute of Pathology provided in depth classification and detailed description of the radiological and clinicopathological features of each of these individual lesions. This by far is the most detailed study on the subject in the English literature with thorough descriptions of each specific entity.

According to the histology of polyp,[31] the two types are: (1) benign neoplastic polyps, which include epithelial, adenoma, papillary, adenoma, nonpapillary, supporting tissues, hemangioma, lipoma, leiomyoma, granular cell tumor, hyperplasia, adenomatous, adenomyomatous, heterotropia, gastric mucosa, intestinal mucosa, pancreas, liver, polyp, inflammatory, cholesterol, miscellaneous, fibroxanthogranulomatous inflammation, parasitic infection, others; and (2) malignant polyps, which include adenocarcinoma, miscellaneous, mucinous cystadenoma, squamous cell carcinoma, and adenoacanthoma.

Most gallbladder polyps are benign nonneoplastic lesions that only rarely cause symptoms. The most common type of polyps in the gallbladders is the cholesterol polyp. Adenomas or adenomyomatous polyps are very rare and were discovered in only $0.4 \%$ patients who underwent cholecystectomy for gallbladder disease.[]]

\section{Gallbladder adenomas}

Although adenomas are benign polyps, they can exhibit premalignant behavior. These lesions are habitually pedunculated single lesions and may be associated with gallstones or chronic cholecystitis. Adenomas account for about four premalignant of gallbladder polyps and are considered neoplastic. They range in size from 5 to $20 \mathrm{~mm}$ and are generally solitary.[1]

Gallbladder adenomas are rare, but well-documented benign epithelial tumors. They are usually found incidentally in cholecystectomy specimens or during preoperative imaging studies. Although they are usually asymptomatic, they may present as a result of associated symptomatic gallstones, or from cystic duct obstruction due to large adenoma. Adenomas may be sessile, pedunculated, or just polypoid projections, and most are accompanied by gallstones. Histologically, they can be tubular, papillary, or tubulopapillary. The tubular type is 
most prevalent and is composed of pyloric or intestinal type glands. These adenomas often coexist with hyperplastic and metaplastic lesions, may contain heterogeneous cell populations, and may show a wide range of morphologic patterns that further complicate the histologic interpretation.[7]

\section{Adenomyomatosis}

Adenomyomatosis, a noninflammatory gallbladder alteration, occurs in middle age patients, and the incidence increases with age. Originally depicted as a benign finding, it is currently identified as a precancerous lesion, and cancer cases associated with areas of adenomyomatosis have been reported [014x-6].[6] Adenomyomatosis accounts for up to $25 \%$ of gallbladder polyps and usually localizes to the gallbladder fundus appearing as a solitary polyp. $[\underline{8}, \underline{33}]$

Go to:

\section{Prediction Of Malignancy}

Numerous studies have attempted to define characteristics which increase the likelihood that a given gallbladder polyp may be malignant.[]] Some studies have revealed that age, presence of diabetes mellitus,[34] size of polyp, gallstones, solitary polyp, and symptomatic polyp[35] are important factors for malignancy.

Many studies have demonstrated that malignant gallbladder polyps are significantly more common in patients aged over $50 .[1, \underline{3}, \underline{8}, \underline{34}, \underline{35}]$ Polyp size has long been noted to be an important factor. Gallbladder polyps larger than $1.5 \mathrm{~cm}$, especially in solitary sessile hypoechogenic polyps, are associated with a risk of malignancy. $[1, \underline{3}, \underline{4}, \underline{8}, \underline{34}, \underline{36}]$

Generally, polyps that are smaller than $1 \mathrm{~cm}$ and are asymptomatic are monitored for $6-12$ months with control ultrasound scans, in order to detect any rapid growth. However, some studies have demonstrated that the polyp's diameter alone is not a safe exclusion criteria for neoplasm, $[2,32]$ and therefore, recommend strongly consideration for cholecystectomy for any polyp greater than $6 \mathrm{~mm} \cdot[9,37]$

\section{Correlation between ultrasonography and histopathology}

To analyze the ultrasonographic and histopathologic findings of patients operated due to gallbladder polyps, the records of patients with ultrasonographic diagnosis of gallbladder polyp and that underwent cholecystectomy in a 13 years period were reviewed, collecting their demographic, ultrasonographic, and histopathological data. One hundred and twenty three patients were operated. All were single and larger than 10 $\mathrm{mm}$. It was found that there is a significant correlation between ultrasonographic and histopathological polyp size determination. Polyp size was also a predictor of the presence of adenoma. It was concluded that there is a good correlation between the size of the gallbladder polyp in US and the size in the histopathology report.[38]

\section{Shape of polyps}

Sessile morphology is one of the important factors that suggest malignancy.[3] The sessile morphology was more dominant in the malignant patients. One of the possible explanations for frequent sessile morphology in malignant polypoid lesions of the gallbladder may be that most gallbladder cancers arise in situ from flat, dysplastic epithelium.[3] The patients with sessile polyps have a higher prevalence of malignancy than do the patients with pedunculated polyps, and the sessile carcinomas are rare at a more advanced stage than the pedunculated carcinomas.[14]

\section{Number of polyps}

The neoplastic polyps tended to be solitary, whereas cholesterol polyps were typically multiple. Single lesion was significantly more frequent in malignant.[3] However, a study includinig 1558 patients with gallbladder solitary polyps did not have a higher risk of neoplastic polyps than multiple polyps.[4]

\section{Tumor with regard to follow-up in time}

One study aimed to evaluate the malignant risk of gallbladder polyps, 1558 patients with gallbladder polyps were diagnosed and followed with US. It took 7 years to notice the growth of one of neoplastic polyps. Therefore, a long period of follow-up to detect changes in gallbladder polyps was recommended. Small polyps have a risk of malignancy, careful long-term follow-up of gallbladder polyps will help detect and treat early gallbladder cancer.[]

\section{Presence of gallstones}

The presence of stones or biliary sludge appears to be a risk factor. However, there are two concerns to consider before making gallbladder polyps with gallstones and indication for cholecystectomy. First, its associated risk is not as great as polyp size. When we consider both polyp size and gallstones, stones alone show only ambiguous significance, and the presence of stone is not very useful in predicting small neoplastic polyps. 
Second, there is a technical problem, as the presence of gallstones hinders the precise evaluation of gallbladder polyps with ultrasound. Thus, gallbladder polyp has to be evaluated more carefully in the presence of gallstones because gallstones are both a risk factor and cause for interference.[4]

\section{Tumor markers}

Serum CEA and CA 19-9 have little role in differentiating benign and malignancy. The impracticality of the tumor markers in differential diagnosis may be explained by the fact that most of the malignant polyps in polypoid lesions of the gallbladder are early gallbladder cancers.[]]

\section{Primary sclerosing cholangitis}

Primary sclerosing cholangitis is one of the risk factors for malignancy in a gallbladder polyp. Any gallbladder polyp, regardless of size, in a patient with primary sclerosing cholangitis should be considered for cholecystectomy. Solitary sessile polyps greater than $10 \mathrm{~mm}$ in patients over age 50 should be considered for cholecystectomy, particularly in patients with cholelithiasis and primary sclerosing cholangitis.[ㄱ-9,, 39$]$ Although gallbladder polyps are common and are usually benign in the general population, they are often malignant in primary sclerosing cholangitis. Therefore, regardless of size, any gallbladder polyps in a patient with primary sclerosing cholangitis should be considered for cholecystectomy.[40] If a cholecystectomy is not performed, careful follow-up is warranted.[41]

\section{Indications For Surgery And Follow Up}

Any gallbladder polyp that is felt to be symptomatic should be removed. In patients at risk for malignancy, a polyp of $6 \mathrm{~mm}$ or greater should likely be resected. Patients without risk factors are good candidates for EUS for further evaluation. Those polyps that are considered high risk by EUS criteria should be considered for resection.

The cholecystectomy should only be undertaken in cases where there are clinical signs of gallbladder polyps, polyps with diameters greater than $10 \mathrm{~mm}$, fast-growing polyps, sessile polyps or wide-based polyps, polyps with long pedicles, patient aged over 50, concurrent gallstones, polyps of the gallbladder in fundibulum or abnormal gallbladder wall ultrasound.[1] The surgery of choice is laparoscopic cholecystectomy. A gallbladder polyp greater than $18 \mathrm{~mm}$ in size has a high likelihood of being an advanced cancer; it should be removed with open cholecystectomy, partial liver resection, and possible lymph node dissection.[8]

Gallbladder polyps that are not resected should be followed-up with serial ultrasound examinations. Clear guidelines on a screening interval are so far not available, even though a screening interval of every 6-12 months has been documented.[8] It would be more efficient to make a flexible and tailored follow-up plan or treatment plan for gallbladder polyps based on scientifically found signs rather than fixed or inflexible guidelines.[10]

\section{Methods}

This paper includes an institutional study of patients with pre-operative diagnosis of gall bladder polyp. During a period of 6 years from Jan 2008 to December 2013 twenty six patients were operated for GB polyp by laparoscopic cholecystectomy method. Patients were selected as per the inclusion and exclusion criteria mentioned in the abstract. The study was conducted at INDIRA GANDHI GOVERNMENT MEDICAL COLLEGE, NAGPUR. Patients were followed up for a period of one year post cholecystectomy. A thorough analysis with clinicopathological correlation of all the cases was performed. The study is a prospective type of study in which newly diagnosed cases were included. The imaging modalities used were ultrasonography and computed tomography. Histological confirmation of all the operated cases was performed.

\section{Result}

During the study period 26 cases were operated with a pre-operative diagnosis of GB polyp. The mean age of the patients was found to be 45.5 years and there were 20 males and 6 females as cases. Symptoms of the patients were as follows:- Right hypochondrium pain, dyspepsia, vomitting, two patients had acute cholecystitis features and epigastric discomfort. All the patients underwent laparoscopic cholecystectomy. USG achieved $100 \%$ sensitivity and 4 patients had CT scan also. The mean diameter of the polyps was $6 \mathrm{~mm}$. Out of 26 polyps 21 were cholesterol polyps, 4 were hyperplastic polyps and 1 was found to be an adenoma. After a follow up of 1 year only 2 patients were found to have Right Hypochondrium pain and rest of the patients were completely normal. 


\section{Clinical Recommendations/ Discussion}

If there are signs of malignancy, the patient should have a cholecystectomy in a hospital where a prompt liver resection could be performed. In doubtful cases, a laparoscopic approached may be tried, and a cholecystectomy performed if there are no signs of growth to the serosa at laparoscopy. If there are signs of growth in the serosa, an open approach must be recommended and - if a frozen section shows malignancy - a resection of the nearby liver segments and the tissue in the duodeno-hepatic ligament may be tried. Also if frozen section shows malignancy after laparoscopic cholecystectomy, an enlarged resection must be considered during the same surgical procedure.

In patients with age $\geq 60$ years, sessile polyp morphology, and polyp size $\geq 10 \mathrm{~mm}$, a generous approach to EUS or multislice CT (in the future maybe virtual gallbladder scopy?) for closer characterization should be the normal. In all cases where there are indications of malignancy, a cholecystectomy should be recommended. If there are no signs of malignancy, a follow-up with the same modality is done after 6 months. If by 6 months there is no changes in size, contour, or vascualrity, the follow-up should be individualized - most often a followup after another 12 months could be recommended.

For polyps 6-9 $\mathrm{mm}$ in diameter without signs of malignancy, a new US is recommended after 6 months. If the does not show any significant changes a new US is recommended after another 12 months and if no changes is found than no further imaging studies are made. Gallbladder polyps smaller than $6 \mathrm{~mm}$ are not followed up, if there are no indications of malignancy.

\section{References}

[1]. Matos AS, Baptista HN, Pinheiro C, Martinho F. Gallbladder polyps: How should they be treated and when? Rev Assoc Med Bras. 2010;56:318-21.

[2]. Inui K, Yoshino J, Miyoshi H. Diagnosis of gallbladder tumors. Intern Med. 2011;50:1133-6. PubMed

[3]. Kwon W, Jang JY, Lee SE, Hwang DW, Kim SW. Clinicopathologic features of polypoid lesions of the gallbladder and risk factors of gallbladder cancer. J Korean Med Sci. 2009;24:481-7. [PMC free article]

[4]. Park JY, Hong SP, Kim YJ, Kim HJ, Kim HM, Cho JH, et al. Long-term follow up of gallbladder polyps.J Gastroenterol Hepatol. 2009;24:219-22. [PubMed]

[5]. Kim SY, Lee HS, Lee YS, Chung KW, Jang BK, Chung WJ, et al. Prevalence and risk factors of gallbladder polyp in adults living in Daegu and Gyeongbuk provinces. Korean J Gastroenterol.2006;48:344-50. [PubMed]

[6]. Choi JH, Yun JW, Kim YS, Lee EA, Hwang ST, Cho YK, et al. Pre-operative predictive factors for gallbladder cholesterol polyps using conventional diagnostic imaging. World J Gastroenterol. 2008;14:6831-4. [PMC free article]

[7]. Cantürk Z, Sentürk O, Cantürk NZ, Anik YA. Prevalence and risk factors for gall bladder polyps. East Afr Med J. 2007;84:336-41.

[8]. Gallahan WC, Conway JD. Diagnosis and management of gallbladder polyps. Gastroenterol Clin North Am. 2010;39:359-67.

[9]. Zielinski MD, Atwell TD, Davis PW, Kendrick ML, Que FG. Comparison of surgically resected polypoid lesions of the gallbladder to their pre-operative ultrasound characteristics. J Gastrointest Surg. 2009;13:19-25. [PubMed]

[10]. Yang LP, Yang ZL, Tan XG, Miao XY. Expression of annexin A1 (ANXA1) and A2 (ANXA2) and its significance in benign and malignant lesions of gallbladder. Zhonghua Zhong Liu Za Zhi. 2010;32:595-9.[PubMed]

[11]. Choi SY, Kim TS, Kim HJ, Park JH, Park DI, Cho YK, et al. Is it necessary to perform prophylactic cholecystectomy for asymptomatic subjects with gallbladder polyps and gallstones? J Gastroenterol Hepatol.2010;25:1099-104. [PubMed]

[12]. Kratzer W, Haenle MM, Voegtle A, Mason RA, Akinli AS, Hirschbuehl K, et al. Roemerstein Study Group.Ultrasonographically detected gallbladder polyps: A reason for concern? A seven-year follow-up study. BMC Gastroenterol. 2008;8:41. [PMC free $\underline{\text { article] }}$

[13]. Chattopadhyay D, Lochan R, Balupuri S, Gopinath BR, Wynne KS. Outcome of gall bladder polypoidal lesions detected by transabdominal ultrasound scanning: A nine year experience. World J Gastroenterol.2005;11:2171-3. [PubMed]

[14]. Furukawa H, Kosuge T, Shimada K, Yamamoto J, Kanai Y, Mukai K, et al. Small polypoid lesions of the gallbladder.Differential diagnosis and surgical indications by helical computed tomography. Arch Surg.1998;133:735-9. [PubMed]

[15]. Sun XJ, Shi JS, Han Y, Wang JS, Ren H. Diagnosis and treatment of polypoid lesions of the gallbladder: Report of 194 cases. Hepatobiliary Pancreat Dis. 2004;3:591-4. [PubMed]

[16]. Ito H, Hann LE, D’Angelica M, Allen P, Fong Y, Dematteo RP, et al. Polypoid lesions of the gallbladder: Diagnosis and followup. J Am Coll Surg. 2009;208:570-5.

[17]. Damore LJ, 2nd, Cook CH, Fernandez KL, Cunningham J, Ellison EC, Melvin WS. Ultrasonography incorrectly diagnoses gallbladder polyps. Surg Laparosc Endosc Percutan Tech. 2001;11:88-91. [PubMed]

[18]. Stenberg B, Elliott S. Diagnosis of gallbladder problems using three-dimensional ultrasound. Eur Radiol.2010;20:908-14.

[19]. Jang JY, Kim SW, Lee SE, Hwang DW, Kim EJ, Lee JY, et al. Differential diagnostic and staging accuracies of high resolution ultrasonography, endoscopic ultrasonography, and multidetector computed tomography for gallbladder polypoid lesions and gallbladder cancer. Ann Surg. 2009;250:943-9. [PubMed]

[20]. Pasławski M, Krupski W, Złomaniec J. The value of ultrasound harmonic imaging in the diagnostics of gall bladder cholesterol polyps. Ann Univ Mariae Curie Sklodowska Med. 2004;59:293-7.

[21]. Hattori M, Inui K, Yoshino J, Miyoshi H, Okushima K, Nakamura Y, et al. Usefulness of contrast-enhanced ultrasonography in the differential diagnosis of polypoid gallbladder lesions. Nihon Shokakibyo Gakkai Zasshi. 2007;104:790-8. [ubMed]

[22]. Numata K, Oka H, Morimoto M, Sugimori K, Kunisaki R, Nihonmatsu H, et al. Differential diagnosis of gallbladder diseases with contrast-enhanced harmonic gray scale ultrasonography. J Ultrasound Med.2007;26:763-74. [PubMed]

[23]. Cheon YK, Cho WY, Lee TH, Cho YD, Moon JH, Lee JS, et al. Endoscopic ultrasonography does not differentiate neoplastic from non-neoplastic small gallbladder polyps. World J Gastroenterol. 2009;15:2361-6. [PMC free article]

[24]. Sugiyama M, Atomi Y, Yamato T. Endoscopic ultrasonography for differential diagnosis of polypoid gall bladder lesions: Analysis in surgical and follow up series. Gut. 2000;46:250-4. PMC free article

[25]. Azuma T, Yoshikawa T, Araida T, Takasaki K. Differential diagnosis of polypoid lesions of the gallbladder by endoscopic ultrasonography. Am J Surg. 2001;181:65-70. [PubMed] 
[26]. Cho JH, Park JY, Kim YJ, Kim HM, Kim HJ, Hong SP, et al. Hypoechoic foci on EUS are simple and strong predictive factors for neoplastic gallbladder polyps. Gastrointest Endosc. 2009;69:1244-50. [PubMed]

[27]. Choi WB, Lee SK, Kim MH, Seo DW, Kim HJ, Kim DI, et al. A new strategy to predict the neoplastic polyps of the gallbladder based on a scoring system using EUS. Gastroinest Endsc. 2000;52:372-9.[PubMed]

[28]. Sadamoto Y, Oda S, Tanaka M, Harada N, Kubo H, Eguchi T, et al. A useful approach to the differential diagnosis of small polypoid lesions of the gallbladder, utilizing an endoscopic ultrasound scoring system. Endoscopy. 2002;34:959-65. [PubMed]

[29]. Park KW, Kim SH, Choi SH, Lee WJ. Differentiation of nonneoplastic and neoplastic gallbladder polyps $1 \mathrm{~cm}$ or bigger with multidetector row computed tomography. J Comput Assist Tomogr. 2010;34:135-9.

[30]. Irie H, Kamochi N, Nojiri J, Egashira Y, Sasaguri K, Kudo S. High b-value diffusion-weighted MRI in differentiation between benign and malignant polypoid gallbladder lesions. Acta Radiol. 2011;52:236-40.[PubMed]

[31]. Bang S. Natural course and treatment strategy of gallbladder polyp. Korean J Gastroenterol.2009;53:336-40. [PubMed]

[32]. Ilias EJ. Gallbladder polyps: How should they be treated and when? Rev Assoc Med Bras. 2010;56:258-9. [PubMed]

[33]. Owen CC, Bilhartz LE. Gallbladder polyps, cholesterolosis, adenomyomatosis, and acute acalculous cholecystitis. Semin Gastrointest Dis. 2003;14:178-88. [PubMed]

[34]. Cha BH, Hwang JH, Lee SH, Kim JE, Cho JY, Kim H, et al. Pre-operative factors that can predict neoplastic polypoid lesions of the gallbladder. World J Gastroenterol. 2011;17:2216-22. PMC free article

[35]. Lee KF, Wong J, Li JC, Lai PB. Polypoid lesions of the gallbladder. Am J Surg. 2004;188:186-90.[PubMed]

[36]. Kianmanesh R, Scaringi S, Castel B, Flamant Y, Msika S. Precancerous lesions of the gallbladder. J Chir (Paris) 2007;144:27886. [PubMed]

[37]. Lee JS, Lee KT, Jung JH, Ok SW, Choi SC, Lee KH, et al. Factors associated with malignancy in gallbladder polyps without gallbladder stone. Korean J Gastroenterol. 2008;52:97-105. [PubMed]

[38]. Escalona A, León F, Bellolio F, Pimentel F, Guajardo M, Gennero R, et al. Gallbladder polyps: Correlation between ultrasonographic and histopathological findings. Rev Med Chil. 2006;134:1237-42.

[39]. Kimura K, Fujita N, Noda Y, Kobayashi G, Ito K. Differential diagnosis of large-sized pedunculated polypoid lesions of the gallbladder by endoscopic ultrasonography: A prospective study. J Gastroenterol.2001;36:619-22. [PubMed]

[40]. Leung UC, Wong PY, Roberts RH, Koea JB. Gall bladder polyps in sclerosing cholangitis: Does the 1 -cm rule apply? ANZ J Surg. 2007;77:355-7. [PubMed]

[41]. Buckles DC, Lindor KD, Larusso NF, Petrovic LM, Gores GJ. In primary sclerosing cholangitis, gallbladder polyps are frequently malignant. Am J Gastroenterol. 2002;97:1138-42 